\title{
Efficacy and safety of fondaparinux for the prevention of venous thromboembolism in older acute medical patients: randomised placebo controlled trial
}

Alexander T Cohen, Bruce L Davidson, Alexander S Gallus, Michael R Lassen, Martin H Prins, Witold Tomkowski, Alexander G G Turpie, Jan F M Egberts, and Anthonie W A Lensing for the ARTEMIS Investigators

\begin{abstract}
Objective To determine the efficacy and safety of the anticoagulant fondaparinux in older acute medical inpatients at moderate to high risk of venous thromboembolism.

Design Double blind randomised placebo controlled trial.

Setting 35 centres in eight countries.

Participants 849 medical patients aged 60 or more admitted to hospital for congestive heart failure, acute respiratory illness in the presence of chronic lung disease, or acute infectious or inflammatory disease and expected to remain in bed for at least four days.

Interventions $2.5 \mathrm{mg}$ fondaparinux or placebo subcutaneously once daily for six to 14 days.

Outcome measure The primary efficacy outcome was venous thromboembolism detected by routine bilateral venography along with symptomatic venous thromboembolism up to day 15. Secondary outcomes were bleeding and death. Patients were followed up at one month.

Results 425 patients in the fondaparinux group and 414 patients in the placebo group were evaluable for safety analysis (10 were not treated). 644 patients $(75.9 \%$ ) were available for the primary efficacy analysis. Venous thrombembolism was detected in 5.6\% (18/321) of patients treated with fondaparinux and $10.5 \%(34 / 323)$ of patients given placebo, a relative risk reduction of $46.7 \%$ (95\% confidence interval $7.7 \%$ to $69.3 \%$ ). Symptomatic venous thromboembolism occurred in five patients in the placebo group and none in the fondaparinux group $(\mathrm{P}=0.029)$. Major bleeding occurred in one patient $(0.2 \%)$ in each group. At the end of follow-up, 14 patients in the fondaparinux group (3.3\%) and 25 in the placebo group (6.0\%) had died.

Conclusion Fondaparinux is effective in the prevention of asymptomatic and symptomatic venous thromboembolic events in older acute medical patients. The frequency of major bleeding was similar for both fondaparinux and placebo treated patients.
\end{abstract}

\section{Introduction}

Many acutely ill medical patients, such as those with congestive heart failure, respiratory illness, and infectious or inflammatory disease, are potentially at risk of venous thromboembolism. ${ }^{1-5}$ Most patients who die from pulmonary embolism as a complication of being admitted to hospital are medical patients. ${ }^{6}$ Despite these observations, uncertainty remains about the baseline risk for venous thromboembolism as well as the effectiveness of thromboprophylaxis in these patients. This uncertainty has limited the systematic application of thromboprophylaxis in this population. ${ }^{7-11}$ So far only one placebo controlled study has been reported that used bilateral venography to evaluate the presence of venous thromboembolism. ${ }^{12}$

Fondaparinux, a synthetic, selective inhibitor of factor Xa, effectively reduces postoperative venous thromboembolism after orthopaedic surgery. ${ }^{13}$ We carried out a placebo controlled study of fondaparinux in acute medical patients to determine its efficacy and to better document and understand the risk for thrombosis in this large patient group.

\section{Methods}

We considered for inclusion in our study acutely ill patients with congestive heart failure class III/IV (New York Heart Association classification), acute respiratory illness in the presence of chronic lung disease, or clinically diagnosed acute infections or inflammatory disorders such as arthritis, connective tissue diseases, or inflammatory bowel disease. Patients had to be at least 60 years old and expected to remain in bed for at least four days.

Exclusion criteria were a high risk for bleeding; acute bacterial endocarditis; cerebral metastasis; recent haemorrhagic or ischaemic stroke; brain, spinal, or ophthalmological surgery; an indwelling intrathecal or epidural catheter; a serum creatinine level $>180 \mu \mathrm{mol} / \mathrm{l}$ in a well hydrated patient; documented hypersensitivity to contrast media; anticipated intubation for more than 24 hours; use of antithrombotics within 48 hours before randomisation; an indication for anticoagulant prophylaxis or therapy; or life expectancy of less than one month. Written informed consent was obtained from the patients before randomisation.

\section{Study design}

Patients were randomly assigned by investigators to receive once daily subcutaneous injections of either $2.5 \mathrm{mg}$ fondaparinux sodium (Arixtra; GlaxoSmithKline) or placebo, within 48 hours of admission to hospital. Randomisation was carried out using a predefined central randomisation list, balanced in blocks of four. The code was revealed to the investigators on completion of the study.

ARTEMIS group members are on bmj.com 


\section{Research}

Treatment was scheduled until days 6 to 14, and the primary efficacy outcome was deep vein thrombosis detected by routine bilateral venography on days 6 to 15 along with symptomatic venous thromboembolism up to day 15 . Patients were examined for deep vein thrombosis by routine bilateral ascending venography of the legs between days 6 and 15 and no more than one calendar day after the last administration of study drug, or earlier if deep vein thrombosis was clinically suspected. Symptomatic pulmonary embolism was to be confirmed by a high probability lung scan, pulmonary angiography, or helical computed tomography, or at autopsy. ${ }^{14}$ In the absence of autopsy, death was classified due to pulmonary embolism if it occurred suddenly and was otherwise unexplained. Patients were followed up for one month: venous thromboembolism, bleeding, or death was recorded. Extended prophylaxis was at the discretion of the investigators.

\section{Study drugs}

Study drugs were provided in identical boxes, consisting of 15 prefilled single dose syringes, each containing either active drug ( $2.5 \mathrm{mg}$ fondaparinux sodium in $0.5 \mathrm{ml}$ saline) or placebo $(0.5 \mathrm{ml}$ isotonic saline). Investigators were blinded to treatment assignment for the duration of the study.

The use of aspirin or non-steroidal anti-inflammatory drugs was discouraged. Graduated compression stockings and physiotherapy were allowed.

\section{Outcome measures}

The primary efficacy outcome was the composite of deep vein thrombosis detected by routine bilateral venography on days 6 to 15 , and symptomatic venous thromboembolism up to day 15 .

The primary safety outcome was major bleeding during treatment and two days thereafter and was defined as fatal bleeding, bleeding in a critical location, bleeding leading to surgical intervention, or overt bleeding associated with a drop in haemoglobin concentration of $\geq 20 \mathrm{~g} / \mathrm{l}$ or leading to transfusion of two or more units of red blood cells. Secondary safety outcomes were death and minor bleeding. Minor bleeding was defined as clinically relevant overt bleeding not meeting the criteria for major bleeding.

Efficacy and safety outcomes were adjudicated by a central independent committee whose members were unaware of treatment assignment. Accumulated safety data were regularly reviewed by an independent committee.

\section{Statistical analysis}

On the basis of the single reported placebo controlled study that used venography, we expected a venous thromboembolism rate in the placebo group of between $10 \%$ and $14 \% .^{12}$ We calculated the sample size using a two sided type one error of $5 \%$, a power of $85 \%$, an incidence of venous thromboembolism of $12 \%$ in the placebo group, and an assumed risk reduction of $60 \%$. Based on the expected $30 \%$ of patients with no or inadequate venograms, our study required at least 800 patients.

The primary efficacy analysis was carried out on all randomised patients with an available efficacy assessment up to day 15 . The safety analyses included all patients who had received at least one dose of study drug. We carried out the analyses using a two sided Fisher's exact test and considered a $\mathrm{P}$ value of $<0.05$ as statistically significant. We calculated relative risk reductions, with $95 \%$ confidence intervals. Incidences of the primary efficacy outcome were also calculated according to predefined categorical covariates (congestive heart failure, acute respiratory illness, or acute infectious or inflammatory disease separately). We compared survival rates using the log rank test.

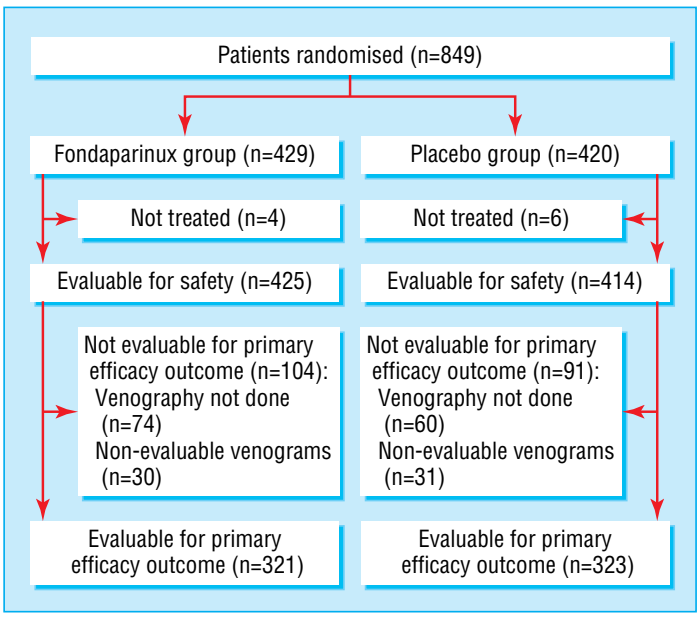

Fig 1 Flow of patients through trial

\section{Results}

Between March 2002 and January 2003, 849 patients from 35 centres in eight countries were randomised to our study (fig 1). Four patients in the fondaparinux group and six in the placebo group were not treated. All treated patients received the allocated study treatment. Thus 425 patients in the fondaparinux group and 414 in the placebo group were evaluable for safety analysis. Treatment lasted for a median of 7 days (range 1-15 days) in the fondaparinux group and 7 days (range 1-13 days) in the placebo group. A total of 644 patients $(75.9 \%)$ were available for the primary efficacy analysis. Reasons for ineligibility for this analysis were failure to obtain a venogram (16.0\%) or an inadequate venogram $(7.3 \%)$. Baseline characteristics were similar between the two groups (table 1).

\section{Incidence of venous thromboembolism}

Overall, $5.6 \%(18 / 321)$ of patients in the fondaparinux group and $10.5 \%(34 / 323)$ of patients in the placebo group had venous thrombembolism detected by routine bilateral venography and symptomatic venous thrombembolism up to day 15 (relative risk reduction $46.7 \%$, 95\% confidence interval $7.7 \%$ to $69.3 \%$,

Table 1 Baseline characteristics of older medical patients randomised to the anticoagulant fondaparinux or placebo. Values are numbers (percentages) unless stated otherwise

\begin{tabular}{|c|c|c|}
\hline Characteristic & $\begin{array}{l}\text { Fondaparinux group } \\
(\mathrm{n}=429)\end{array}$ & Placebo group $(n=420)$ \\
\hline Mean (SD) age (years), range & 75.0 (8.3), 60 to 93 & 74.4 (8.3), 53 to $96^{*}$ \\
\hline Men & $174(40.6)$ & $186(44.3)$ \\
\hline Women & $255(59.4)$ & $234(55.7)$ \\
\hline Mean (SD) weight $(\mathrm{kg})$, range & 70.1 (15.2), 32 to 111 & 70.1 (16.8), 35 to 150 \\
\hline Mean (SD) body mass index & $25.9(5.5)$ & $25.8(5.7)$ \\
\hline \multicolumn{3}{|l|}{ Reason for admission to hospital: } \\
\hline Congestive heart failure (class III/IV) $\dagger$ & $106(24.7)$ & $106(25.2)$ \\
\hline Acute respiratory disease & $74(17.2)$ & $93(22.1)$ \\
\hline $\begin{array}{l}\text { Acute infectious or inflammatory } \\
\text { disease }\end{array}$ & $107(24.9)$ & $107(25.5)$ \\
\hline More than one reason & $142(33.1)$ & $114(27.1)$ \\
\hline \multicolumn{3}{|l|}{ Risk factors for venous thrombembolism: } \\
\hline Age $\geq 75$ years & $223(52.0)$ & $216(51.4)$ \\
\hline History of venous thrombembolism $\ddagger$ & $18(4.2)$ & $21(5.0)$ \\
\hline Previous or current cancer & $62(14.5)$ & $69(16.4)$ \\
\hline
\end{tabular}

*One patient recruited out of prespecified range in placebo group (enrolled at age 53) was not included in primary efficacy population.

†According to New York Heart Association.

fDeep vein thrombosis or pulmonary embolism. 
Table 2 Venous thromboembolic events in older ( $\geq 60$ years) medical patients randomised to the anticoagulant fondaparinux or placebo. Values are numbers unless stated otherwise

\begin{tabular}{lcc} 
Primary efficacy outcome & Fondaparinux group & Placebo group \\
\hline Venous thromboembolic events: & & \\
\hline Any & 18 & 29 \\
\hline Proximal deep vein thrombosis & 5 & 7 \\
\hline Distal deep vein thrombosis & 13 & 22 \\
\hline Symptomatic deep vein thrombosis & 0 & 0 \\
\hline Non-fatal pulmonary embolism & 0 & 0 \\
\hline Fatal pulmonary embolism & 0 & 5 \\
\hline Total No (\%) & $18 / 321(5.6)$ & $34 / 323(10.5)$ \\
\hline No (\%) of any symptomatic venous & & \\
thrombembolism up to day 32: & 0 & 0 \\
\hline Symptomatic deep vein thrombosis & 1 & 4 \\
\hline Non-fatal pulmonary embolism & 3 & 7 \\
\hline Fatal pulmonary embolism & $4 / 429(1)$ & $11 / 420$ \\
\hline Total No (\%) & $3)$ \\
\hline
\end{tabular}

$\mathrm{P}=$ 0.029: table 2). Consistent reductions were shown in the incidence of total, proximal, and distal deep vein thrombosis. Five fatal cases of pulmonary emboli occurred during the study, all in the placebo group. Two of the five were confirmed by autopsy, the others were assumed to be due to pulmonary emboli, as no other plausible cause was found.

The incidence of venous thrombembolism on venography and symptomatic venous thromboembolism was reduced in fondaparinux recipients with only congestive heart failure from $12.2 \%(10 / 82)$ to $9.0 \%(7 / 78)$, only acute respiratory illness from $5.5 \%(4 / 73)$ to $1.6 \%(1 / 63)$, only acute infectious or inflammatory disease from $11.4 \%(10 / 88)$ to $5.2 \%(4 / 77)$, and in patients with more than one reason for admission to hospital from $12.5 \%(10 / 80)$ to $5.8 \%(6 / 103)$.

Ten additional case of pulmonary embolism occurred during follow-up (four in the fondaparinux group). Of these, five were fatal (three in the fondaparinux group).

\section{Safety outcomes}

Major bleeding occurred in one patient $(0.2 \%)$ in each group during the study, both related to overt bleeding and a drop in haemoglobin concentration of $\geq 20 \mathrm{~g} / 1$ or transfusion of $\geq 2$ units of blood (table 3). Minor bleeds were observed in 11 patients $(2.6 \%)$ in the fondaparinux group and four $(1.0 \%)$ in the placebo group.

At one month, $14(3.3 \%)$ patients in the fondaparinux group and $25(6.0 \%)$ in the placebo group had died $(\mathrm{P}=0.06)$ (fig 2 , table 4).

\section{Discussion}

Daily subcutaneous injections of $2.5 \mathrm{mg}$ fondaparinux for 6-14 days almost halved the rate of venous thromboembolism in older

Table 3 Bleeding complications during study treatment of older ( $\geq 60$ years) medical patients randomised to the anticoagulant fondaparinux or placebo

\begin{tabular}{lcc} 
Bleeding complication & $\begin{array}{c}\text { Fondaparinux group } \\
(\mathbf{n}=\mathbf{4 2 5})\end{array}$ & $\begin{array}{c}\text { Placebo group } \\
(\mathbf{n}=\mathbf{4 1 4})\end{array}$ \\
\hline Major bleeding: & & \\
\hline Fatal & 0 & 0 \\
\hline Requiring surgical intervention & 0 & 0 \\
\hline In a critical location & 1 & 0 \\
\hline $\begin{array}{l}\text { Overt bleeding plus a decrease in haemoglobin } \\
\text { concentration } \geq 20 \mathrm{~g} / \mathrm{L}(<48 \text { hours) or transfusion } \\
\text { of } \geq 2 \text { units }\end{array}$ & & 1 \\
\hline Total No $(\%)$ & $1(0.2)$ & $1(0.2)$ \\
\hline Minor bleeding & $11(2.6)$ & $4(1.0)$ \\
\hline
\end{tabular}

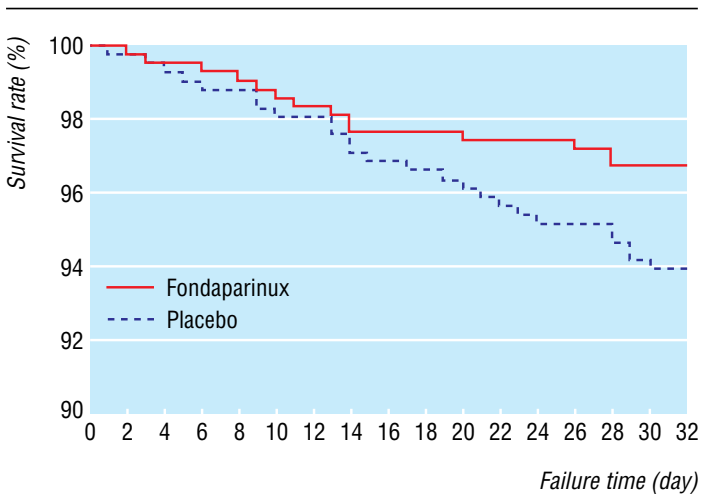

Fig 2 Survival estimates of older ( $\geq 60$ years) acute medical patients treated with the anticoagulant fondaparinux or placebo. Log rank test on difference, $\mathrm{P}=0.06$

( $\geq 60$ years) acute medical patients admitted to hospital and requiring bed rest for heart, lung, or infectious or inflammatory disorders. No patients in the fondaparinux group and five in the placebo group had symptomatic fatal or non-fatal pulmonary embolism $(\mathrm{P}=0.029)$. This reduction in venous thromboembolism was achieved with a minimal risk of major bleeding complications.

The relative risk reduction with fondaparinux in this double blind trial is consistent with results obtained by the high dosage low molecular weight heparin group in the placebo controlled study that used venography. ${ }^{12}$ These results add to the efficacy data supporting the general applicability of fondaparinux in the prevention as well as treatment of venous thromboembolism..$^{13}$

The two aspects of our trial design that required greatest consideration were the clinical selection criteria for patient eligibility and the choice of placebo as comparator for fondaparinux. Older medical inpatients are a clinically diverse group. Thrombosis risks are well known after surgical interventions, but when our study was designed few recent studies had evaluated this risk in older people admitted to hospital with other acute or chronic conditions. Guidelines are available on prophylaxis in high risk inpatients but a need for universal prophylaxis has not been shown. ${ }^{15}$ No simple and easily applied selection criteria exist, and audits of general medical inpatients indicate that thrombosis prevention is not systematically applied or well grounded on risk factor assessment. ${ }^{71016}$

Clinical events are not rare in medical patients (around 10\% of deaths are due to pulmonary embolism ${ }^{17}$ ) but are in thromboprophylaxis studies. ${ }^{12}{ }^{19}$ Clinical events are rare in this type of study as venogaphy is a sensitive and specific screening tool (more so than duplex Doppler ultrasound).

Venography is able to diagnose a higher frequency of deep vein thrombosis and smaller thrombi (presumably at an earlier stage). This technique is the ideal choice. Earlier and more accurate diagnosis has advantages for patients, with therapy starting

Table 4 Mortality up to day 32 in older ( $\geq 60$ years) medical patients randomised to the anticoagulant fondaparinux or placebo

\begin{tabular}{lcc} 
Variable & $\begin{array}{c}\text { Fondaparinux group } \\
(\mathbf{n}=\mathbf{4 2 5})\end{array}$ & $\begin{array}{c}\text { Placebo group } \\
(\mathbf{n = 4 1 4 )}\end{array}$ \\
\hline Death from any cause: & & \\
\hline Fatal pulmonary embolism & 3 & 7 \\
\hline Fatal bleeding ${ }^{\star}$ & 2 & 1 \\
\hline Other causes & 9 & 17 \\
\hline Total No (\%) & $14(3)$ & $25(6)$ \\
\hline
\end{tabular}

*None occurred during study treatment. 


\section{What is already known on this topic}

Most patients who die from pulmonary embolism as a complication of being admitted to hospital are medical patients

The effectiveness of thromboprophylaxis in these patients is uncertain

\section{What this study adds}

Fondaparinux is effective in the prevention of venous thromboembolism in acutely ill older ( $\geq 60$ years) medical patients

before disease progression. As a result, clinical venous thrombembolism is rare in patients in whom venography studies are undertaken. We have concluded in favour of fondaparinux for two reasons, firstly because the surrogate end point of venographic deep vein thrombosis is strongly correlated to clinical deep vein thrombosis, clinical pulmonary embolism, ${ }^{16}$ and fatal pulmonary embolism. ${ }^{20}$ Secondly, we found a significant reduction in the clinical secondary end point of fatal pulmonary embolism. Similar conclusions have been made in studies in both this area and other areas on the basis of surrogate end points ${ }^{16}{ }^{20}$ and by the American College of Chest Physicians. ${ }^{15}$ We do not think that our conclusions are unjustified on the basis of our findings.

We believed that our study could best contribute to the improvement of clinical practice if we applied simple and easily generalisable patient selection criteria to general medical inpatients (age $\geq 60$ years, recent and continuing bed rest, and presence of a heart, lung, or infectious or inflammatory disorder), without further risk factor assessment. Since these criteria differed from those applied by others, our trial depended on a placebo group to measure baseline risk and risk reduction by active therapy. ${ }^{12} 16$ Investigators excluded patients prescribed or thought to need thromboprophylaxis. This might explain why the $10.5 \%$ thromboembolism rate at baseline observed was below previous levels of 20\% in early studies and $15 \%$ in the placebo controlled trial. ${ }^{12}{ }^{15}$ Therefore our simple case selection criteria defined a group of patients at moderate risk in which prophylaxis is clearly effective and justified. Hence we believe that our results can be generalised to all medical inpatients with these diagnoses. Moreover, these criteria can be easily applied and audited.

Our results are likely to be valid, because of randomised treatment assignment, the use of a double blind design, the objective diagnosis of venous thromboembolism, and the central adjudication of study outcomes while unaware of treatment assignment. These features minimised the potential for bias.

Two thirds of the clinically apparent events and half of the fatal pulmonary emboli were observed after the initial 6-14 day study period. This is similar to the observation in one trial and supports the need to evaluate extended prophylaxis in medical patients. ${ }^{12}$ Our results when added to those obtained with low molecular weight heparins ${ }^{12}{ }^{15}$ establish the value of thromboprophylaxis in older medical inpatients at moderate to high risk of venous thromboembolism. The challenge now is to translate shown efficacy into effectiveness through local clinical practice guidelines, practice audit, and practice feedback.

Contributors: All authors developed the protocol, interpreted the data, and wrote the manuscript. MHP supervised the statistics. JFME and AWAL ensured the protocol adhered to guidelines issued by the European Agency for the Evaluation of Medicinal Products and Food and Drug Administration, recruited the clinical investigators who treated the patients, and gathered data. ATC is guarantor for the paper.

Funding: Sanofi-Synthelabo (France) and NV Organon (Netherlands) sponsored the study and carried out on-site monitoring of all participants. The steering committee had the final responsibility for the study protocol, case report forms, statistical analysis plan, progress of the study and analysis, as well as the reporting of the data. The sponsors had an opportunity to comment on the manuscripts before submission, but the final version was the sole responsibility of the authors.

Competing interests: ATC, BLD, ASG, MRL, WT, and AGGT participated as investigators, consultants, or both for NV Organon and Sanofi-Synthelabo. JFME and AWAL are employees of NV Organon. BLD has served as an investigator or consultant for AstraZeneca, Bristol-Myers Squibb, Boehringer Ingelheim, and Pharmacia. ASG has served as an investigator, consultant, or advisory board member for Bristol-Myers Squibb, AstraZeneca, Aventis, Bayer, and Progen. MRL has served as an investigator and advisory board member for AstraZeneca, Britol-Myers Squibb, Mitsubishi Pharma Europe, Yamanouchi Pharma, and Bayer. AGGT is a consultant for BristolMyers Squibb.

Ethical approval: The institutional review boards of each centre approved the protocol.

1 Anderson FA Jr, Wheeler HB, Goldberg RJ, Hosmer DW, Patwardhan NA, Jovanovic B, et al. A population-based perspective of the hospital incidence and case-fatality rates of deep vein thrombosis and pulmonary embolism. The Worcester DVT study. Arch Intern Med 1991;151:933-8.

2 Kniffin WD Jr, Baron JA, Barrett J, Birkmeyer JD, Anderson FA Jr. The epidemiology of diagnosed pulmonary embolism and deep venous thrombosis in the elderly. Arch Intern Med 1994;154:861-6.

3 Weinmann EE, Salzman EW. Deep-vein thrombosis. N Engl J Med 1994:331:1630-41.

4 Heit JA, O'Fallon WM, Petterson TM, Lohse CM, Silverstein MD, Mohr DN, et al. Relative impact of risk factors for deep vein thrombosis and pulmonary embolism: a tive impact of risk factors for deep vein thrombosis and
population-based study. Arch Intern Med 2002;162:1245-8.

population-based study. Arch Intern Med 2002;162:1245-8.
5 Baglin TP, White K, Charles A. Fatal pulmonary embolism in hospitalised medical patients. J Clin Pathol 1997;50:609-10.

6 Cohen AT, Edmondson RA, Phillips MJ, Ward VP, Kakkar VV. The changing pattern of venous thrombembolic disease. Haemostasis 1996;26:65-71.

7 Ageno W, Squizzato A, Ambrosini F, Dentali F, Marchesi C, Mera V, et al. Thrombosis prophylaxis in medical patients: a retrospective review of clinical practice patterns. Haematologica 2002;87:746-50.

8 Alikhan R, Wilmott R, Agrawal S, Desouza N, Gera A, Zaw H, et al. Use of thromboprophylaxis in nonsurgical patients: survey of a London teaching hospital. Blood 2001:98:271a (abstract 1142).

9 Cohen AT. Venous thromboembolic disease management of the nonsurgical Cohen AT. Venous thromboembolic disease management of the
moderate- and high-risk patient. Semin Hematol 2000;37(suppl.5):19-22.

10 Mismetti P, Laporte S. Thromboprophylaxis in acute medical patients: need for an implementation strategy. Haematologica 2002;87:673-5.

11 Hampton KK. Venous thromboembolic risk and prevention in acute medical illness. Int J Clin Pract 2003;57:424-7.

12 Samama MM, Cohen AT, Darmon JY, Desjardins L, Eldor A, Janbon C, et al. A comparison of enoxaparin with placebo for the prevention of venous thromboembolism in acutely ill patients. N Engl J Med 1999;341:793-800.

13 Turpie AGG, Bauer KA, Eriksson BI, Lassen MR. Fondaparinux vs enoxaparin for the prevention of venous thromboembolism in major orthopedic surgery. A meta-analysis of 4 randomized double-blind studies. Arch Intern Med 2002;162:1833-40.

14 The Matisse Investigators. Subcutaneous fondaparinux versus intravenous unfractionThe Matisse Investigators. Subcutaneous fondaparinux versus intravenous unfraction-
ated heparin in the initial treatment of pulmonary embolism. $N$ Engl J Med ated heparin in the

15 Geerts WH, Heit JA, Clagett GP, Pineo GF, Colwell CW, Anderson FA Jr, et al. Prevention of venous thromboembolism. Chest 2001;119:S132-75.

16 Mismetti P, Laporte-Simitsidis S, Tardy B, Cucherat M, Buchmuller A, Juillard-Delsart $\mathrm{D}$, et al. Prevention of venous thromboembolism in internal medicine with unfractionated or low-molecular-weight heparins: a meta-analysis of randomized clinical trials. Thromb Haemost 2000;83:14-9.

17 Lindblad B, Sternby NH, Bergqvist D. Incidence of venous thromboembolism verified by necropsy over 30 years. BMJ 1991;302:709-11.

18 Sandler DA, Martin JF. Autopsy proven pulmonary embolism in hospital patients: are we detecting enough deep vein thrombosis? JR Soc Med 1989;82:203-5.

19 Leizorovicz A, Cohen AT, Turpie AG, Olsson CG, Vaitkus PT, Goldhaber SZ, et al. A Leizorovicz A, Cohen AT, Turpie AG, Olsson CG, Vaitkus PT, Goldhaber SZ, et al. A
randomized placebo controlled trial of dalteparin for the prevention of venous thromrandomized placebo controlled trial of dalteparin for the prevention of venous throm-
boembolism in 3706 acutely ill medical patients: the PREVENT medical boembolism in 3706 acutely ill medical patients: th
thromboprophylaxis study trial. Circulation 2004;110:8749.

20 Collins R, Scrimgeour A, Yusuf S, Peto R. Reduction in fatal pulmonary embolism and venous thrombosis by perioperative administration of subcutaneous heparin. Overview of results of randomised trials in general, orthopaedic, and urologic surgery. N Engl J Med 1988;318:1162-73.

(Accepted 17 November 2005)

doi 10.1136/bmj.38733.466748.7C

Department of Surgery, Guy's, King's, and St Thomas's School of Medicine, London SE5 9PJ

Alexander T Cohen honorary consultant

Pulmonary and Critical Care, University of Washington School of Medicine and Swedish Medical Center, Seattle, WA, USA 
Research

Bruce L Davidson clinical professor of medicine

Department of Haematology, Flinders Medical Centre, Adelaide, SA, Australia Alexander S Gallus professor

Spine Clinic, Department of Clinical Research, Hørsholm, Denmark Michael R Lassen chair

Department of Clinical Epidemiology and Technology Assessment, Academic Hospital Maastricht, Netherlands

Martin H Prins professor of clinical epidemiology

Intensive Care, National TB and Lung Diseases Research Institute, Warsaw, Poland
Witold Tomkowski head of cardiopulmonary intensive care

Department of Medicine, Hamilton Health Sciences, Hamilton, ON, Canada Alexander G G Turpie professor

Clinical Development, NV Organon, Oss, Netherlands Jan F M Egberts research scientist

Clinical Development, NV Organon, Oss, Netherlands; Center for Vascular Medicine, Academic Medical Center, Amsterdam, Netherlands

Anthonie W A Lensing research physician

Correspondence to: A T Cohen alexander.cohen@kcl.ac.uk 